\title{
Skin stretch haptic feedback to convey closure information in anthropomorphic, under-actuated upper limb soft prostheses.
}

\author{
Edoardo Battaglia, Janelle P. Clark, Matteo Bianchi, \\ Manuel G. Catalano, Antonio Bicchi, and Marcia K. O'Malley
}

\begin{abstract}
Restoring hand function in individuals with upper limb loss is a challenging task, made difficult by the complexity of human hands from both a functional and sensory point of view. Users of commercial prostheses, even sophisticated devices, must visually attend to the hand to know its state, since in most cases they are not provided with any direct sensory information. Among the different types of haptic feedback that can be delivered, information on hand opening is particularly likely to reduce the requirement of constant visual attention. In recent years there has been a trend of using underactuated, compliant multi-fingered hands as upper limb prostheses, in part due to their simplicity and ease of use attributed to low degree-of-freedom actuation. The trend towards underactuation encourages the design of one degree of freedom (d.o.f.) haptic devices to provide intuitive sensory feedback from the prosthesis. However, mapping the closure of a multi-d.o.f. prosthetic hand to a simple and intuitive haptic cue is not a trivial task. In this paper we explore the use of a one d.o.f. skin stretch haptic device, the Rice Haptic Rocker, to provide intuitive proprioceptive feedback indicating overall hand closure of an underactuated prosthesis. The benefits and challenges of the system are assessed in multi-tasking and reduced vision scenarios for an object-size discrimination task, in an effort to simulate challenges in daily life, and are compared against the haptic resolution of the device using the Just Noticeable Difference. Finally, an evaluation done with a prosthesis user, in the form of a truncated version of the AM-ULA, shows possible benefits of the addition of haptic feedback in tasks with reduced visual attention.
\end{abstract}

\section{INTRODUCTION}

L OSING a limb has important consequences on the ability to function in daily activities [1]. It is possible to reproduce hand functionality to some extent through a prosthesis; however, restoring the versatility of a human hand is very challenging, especially for what concerns ease of use and the ability to convey sensory information. Traditionally many of the artificial hands in clinical practice have been either purely cosmetic or body powered [2], i.e. actuated through cables that are mechanically pulled by the user, typically by a harness attached to the shoulder. Body powered prostheses have the advantage of being simple and intrinsically able to partially convey haptic feedback to the user through the actuation; however, they can also suffer from lack of comfort and small grip forces.

In myoelectric prostheses, the hand is actuated by one or more electrical motors controlled by electro-myographic (EMG) signals generated by electrodes placed on the skin over the the user's muscles. This approach has a higher level of technology and potential to offer better comfort and functionality; however, it is more difficult to control for the user [3], [4]. Moreover, myoelectric prostheses lack the inherent haptic feedback offered by body powered prostheses, and require visual attention on the artificial hand at all times during use. This can generate frustration for the user and cause abandonment of the prosthesis, which

- Edoardo Battaglia, Matteo Bianchi and Antonio Bicchi are with Centro di Ricerca "E. Piaggio" and Dipartimento di Ingegneria dell'Informazione, University of Pisa, Pisa, Italy, 56126

E-mail: e.battaglia,matteo.bianchi,bicchi@centropiaggio.unipi.it

- Janelle P. Clark and Marcia K. O'Malley are with the Department of Mechanical Engineering, Rice University, Houston, Texas, 77251 E-mail: janelle.clark, omalleym@rice.edu

- Manuel G. Catalano and Antonio Bicchi are with the Soft Robotics for Human Cooperation and Rehabilitation Research Line, Instituto Italiano di Tecnologia, Via Morego 30, 16163, Genova, Italy
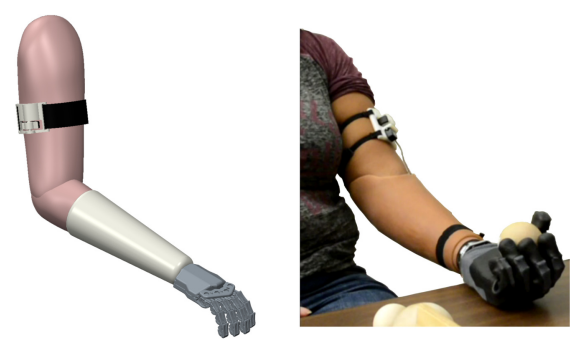

Fig. 1: Envisioned integration of the Rocker and the SoftHand and first application with a prosthesis user.

is observed in many cases and is a serious limitation of myoelectric devices [5]. Indeed, haptic feedback is a desired feature amongst users [1], [6], [7] and has been shown to increase embodiment [8], [9].

Despite this clear opportunity, today the presence of haptic feedback in commercial prosthetic systems is still limited. To try and fill this gap, the research community has proposed different methods to convey haptic information to prosthesis users. Non-invasive solutions typically rely on sensory substitution, with vibrotactile [10], electrotactile [11], force feedback [12], [13] , [14] and skin stretch [15] feedback being delivered to the user by external devices applied to the skin. While different types of feedback devices are useful to convey information on different measurements, simultaneous display of different types of haptic information can also be confusing for the user [16]. For this reason, it is important to focus on conveying information that is most important for task execution.

A common requirement from upper limb prosthesis users is to be able to operate prostheses without constant 
visual attention [6], [17]. In order to make this possible, it is necessary to convey information on the level of opening of the hand, which is commonly known as proprioceptive feedback. If we consider a simple gripper-like prosthesis with a single degree of freedom (d.o.f.), this task is fairly straightforward, as one can simply use the reading from the single motor and map it to a 1-d.o.f. haptic device to convey the information in a complete manner, while keeping the cognitive burden for the user manageable. However, gripper-like artificial hands offer a limited level of dexterity and versatility. For this reason there has been a trend in recent years to develop multi-d.o.f. hands, both for use with robots and as prostheses [18]. It becomes then more challenging to convey proprioceptive information with a single d.o.f. haptic device, as the overall level of opening of such hands is a function of many variables (e.g. joint angles).

One could in principle convey more precise information by using multiple degrees of haptic feedback [19], but this would greatly increase the cognitive burden on the user. For this reason, the use of a one-d.o.f. haptic device still remains preferable. The implementation through a one d.o.f. solution becomes simpler for hands that are under-actuated with a single motor, as the reading of the encoder can be used as a coarse measurement of the overall level of opening of the hand. However, the mapping problem is still not trivial, especially for hands that are compliant, which is a class of devices that is quickly growing in the state of art [18]. Therefore, in this paper we investigate the effectiveness of mapping the closure of a compliant anthropomorphic prosthetic hand, as estimated by the encoder reading of its single actuator, to one d.o.f. haptic feedback.

In [20] we introduced the Rice Haptic Rocker, a device that conveys proprioceptive information to the user through skin stretch, and presented an evaluation of this device with an under-actuated prosthetic hand. In addition to the introduction of the novel device, the main contribution of our preliminary work was that it used a real prosthetic hand to evaluate the value of providing proprioceptive feedback. This is in contrast to the common approach found in the state of art, where testing is done in a virtual reality environment (e.g. [11], [21]-[23]). While this choice allowed us to test the hand under conditions that were closer to a real case scenario, the testing was completed through a passive size discrimination task, where an experimenter placed spheres inside the hand and asked subjects to discriminate by size. Encouraged by the high accuracy observed in our experimental results, in this paper we present further evaluation of the approach, this time under more realistic conditions.

A group of experiments are presented with able-bodied subjects. The first experiment aims to evaluate the effectiveness of proprioceptive feedback when a distraction task is present, inspired by [24], where a secondary acoustic task was presented in parallel with the main haptic task. The second experiment tests the setup in a configuration where the users are actively lifting the hand to reach and grasp objects, which leads to an added level of challenge because the prosthetic hand has multiple degrees of freedom. An evaluation of the device in terms of Just Noticeable Difference and Point of Subjective Equivalence is also shown, which is used to better evaluate the results of the second experiment.

Furthermore, the setup is evaluated with an upper limb prosthesis user performing the clinical evaluation test AMULA [25] both with and without the provision of haptic feedback via the Rice Haptic Rocker, as well as a passive size discrimination task with feedback.

\section{MOTIVATION AND BACKGROUND}

Proprioception is the ability to perceive the position and movements of our own body [26]. This sensing modality allows humans to place parts of their body in space without looking at them, and it plays a role in daily living tasks without our awareness, such as when retrieving something from a shelf without looking at it, or reaching to turn off an alarm clock. The mechanism of proprioception is still not completely understood. While in the past it was thought that joint receptors were primarily responsible for obtaining this information, further studies demonstrated that their role is minor and it is instead the muscle spindles and skin stretch receptors that play a major role in this process [27]. There is evidence that skin stretch is important for proprioception in the hand, where the skin adjacent to finger joints enables sensing of information that could not be obtained through muscle spindles alone [28]. Furthermore, proprioceptive signals are one of the main factors involved in the development of the "sense of agency" or, in other words, the experience of oneself as the agent of one's own actions [29]. This is strictly related to the sense of embodiment, i.e. the perception from the user of the prosthesis as a part of themselves, which can improve functionality and help acceptance [30].

This natural mechanism of proprioception is missing for myoelectric prosthetic users, who rely heavily on vision to know the pose of their artificial hand. For this reason, researchers have been working to introduce proprioceptive feedback in hand prostheses. In [23] it was shown that proprioceptive feedback successfully improves targeting accuracy in nonsighted and, for some tasks, also sighted conditions. Vibrotactile feedback was used in several studies to provide information of the state of a prosthesis [21], [31]. In [11] vibrotactile and electrotactile feedback were used to convey information on hand opening, with a series of eight actuators being activated in sequence, in a task where subjects were asked to match a target opening position that was shown briefly on a screen before disappearing. Vibrotactile feedback improved performance compared to the no haptic feedback condition, while electrotactile feedback was reported as unpleasant for the user and difficult to calibrate. Other studies contradict these conclusions. For example, in the study presented in [32], vibrotactile feedback was not effective, and electrotactile feedback could become feasible in the light of the recent technological developments presented in [33].

Skin stretch has also been investigated as a way to convey proprioceptive feedback. It was mentioned before that there is evidence that skin stretch is an important part of the mechanism that conveys information regarding proprioception of the hand. In this sense, using skin stretch to convey proprioception of prosthetic hands could lead to easier training for upper limb prosthesis users, since the feedback provided is felt in a way that is similar to a natural mechanism found in able-bodied limbs. Indeed, this type of feedback was shown to be an effective way to convey proprioception. In [34], rotational skin stretch was proposed as an alternative to vibrotactile feedback for conveying proprioception, and results showed it to be more effective. Further investigation confirmed the effectiveness 
of this approach [15], [35], which however, owing to the small surface of contact, has the drawback of requiring an adhesive element to be placed as an interface between the skin and the device. In [36], a passive skin stretch device was used to convey information on the opening of a multid.o.f. artificial hand, with results comparable to vibrotactile feedback. This work is especially interesting because of the passive nature of the hardware leading to a lightweight and compact system; however, it relies on a connection to the system of pulleys in the hand, and as such cannot be integrated with a standard prosthesis. Chinello et al. studied combining multiple rockers interacting in a bracelet about the forearm [37] to direct able-bodied subjects in more complex wrist movements, but did not consider prosthetic applications.

Inspired by this line of research, in [20] we introduced the Rice Haptic Rocker, and proposed a different approach to produce skin stretch using a rocking mechanism and a frictional interface that enables movement of the skin without adhesive elements. Studies on proprioceptive feedback typically evaluate performance in virtual reality tasks, with few papers addressing the evaluation of proprioceptive feedback in a physical task (for example in [38] proprioceptive feedback provided information on elbow position, while in [39] a vision based method was used to provide proprioceptive feedback for a hand prosthesis). This is traditionally done to enable an analysis of the effect of proprioceptive feedback under controlled conditions, usually to decouple it from visual feedback. However, when working under such controlled conditions, it is difficult to capture some of the more challenging aspects of conveying proprioception, especially when considering hands with multiple degrees of freedom and intrinsic compliance, as discussed in Section 1.

Indeed, hand opening for a multi-d.o.f. hand cannot be quantified unequivocally with a single number. For a single d.o.f. hand, such as the Ottobock tri-digit hand [40], measurement of the hand opening level is determined by the encoder reading. In contrast, hands with multiple degrees of freedom and actuation (e.g., the BeBionic hand [41]) the hand pose can not be quantified with a single number, as it is related to the position of the digits as commanded by the individual actuation units. A trade-off between the two approaches can be achieved when considering underactuated hands, for which a limited number of motors is used to control many degrees of freedom: in particular when there is only one motor, the encoder position can provide an indication of the overall level of opening of the hand, even if information on the individual digit position is not available. In the following sections we will present a set of experiments that aim to both test our approach under realistic conditions with a single-motor, multi-d.o.f. hand, and evaluate the challenges with conveying proprioceptive feedback in this scenario.

\section{DEVICES AND INTEGRATION}

\subsection{The Pisa/IIT SoftHand}

The Pisa/IIT Softhand design [42] takes inspiration from neuroscience research. It is known that humans control their hands not merely by acting on each of the numerous degrees of freedom, but rather by coordinating and co-activating them in organized motions called synergies [43], [44]. In more recent work [45], soft synergies were introduced, where the synergy serves as a reference position for a virtual hand, and the interaction forces between the hand and a grasped object depend on the stiffness matrix connecting the virtual and real hand position. Compliance and synergy inspiration are built into an artificial hand with 19 d.o.f.s, 4 on each of 4 fingers, and 3 on the thumb; the CAD model and system design are available as an open source project as described in [46]. The fingers are capable of flexion/extension as well as ab/adduction. Traditional revolute joints are employed for $a b / a d d u c t i o n$ of the fingers and at the equivalent of the carpometacarpal joint of the thumb. The remainder of the joints incorporate rolling contact joints with elastic ligaments, which ensure physiologically correct motions when actuated, but easily disengage on impact to allow safe interaction. A single tendon runs though all joints to simultaneously flex and adduct the fingers upon actuation.

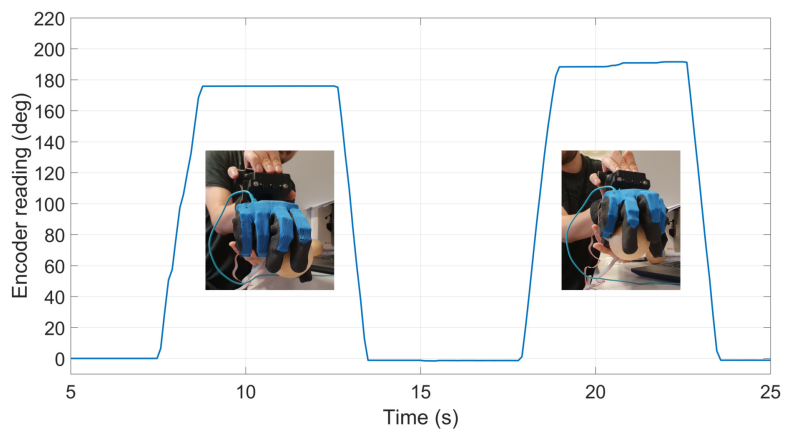

Fig. 2: Encoder reading from the SoftHand as function of time, while grasping the same object in different ways. Since the hand is shaped differently around the object, the readings from the single encoder, measuring the position of the single motor, are different even if the object is the same.

The hand is actuated by one DC motor which moves the fingers on the path of the first synergy as described in [43]. The motor position is measured with a 12 bit, 0.0875 degree resolution magnetic encoder from Austrian Microsystems. In every experiment described in this paper the SoftHand is EMG controlled with the approach described in [42], [47]. Due to the single encoder measurement in the hand, it is intuitive to use the motor position as an indication of hand aperture. In this work, we propose mapping the motor position directly to a 1-d.o.f. haptic feedback device as a synergistic approach to convey the overall level of hand opening. The presence of multiple degrees of freedom and compliance in the hand makes it challenging to convey complete information on the posture of the individual fingers. However, we propose this approach as an intermediate solution between: (a) conveying no information on hand opening, which is the state of the art in commercial myoelectric hands, leaving users unable to know at all if the hand is open or closed without visual attention; and (b), using multiple sensors on the hand to measure the position of every link, which would make it much more expensive, complex, and less robust. Because of its synergistic behavior the SoftHand is an ideal test-bed for an application with a one-d.o.f. haptic device, as we will show in the following sections.

\subsection{The Rocker}

The Rice Haptic Rocker is a wearable device that relies on skin stretch stimulation at the upper arm level to convey 


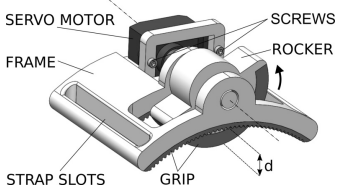

(a) CAD model and parts. The offset $d$ is used to apply pressure of the user's arm.

Fig. 3: The Rice Haptic Rocker provides haptic feedback proportional to the overall level of opening of the SoftHand.

proprioceptive information regarding the prosthetic hand. It has a simple design consisting of a frame, strap, rocker, and servo, as shown in Figure 3a, with a total weight of only $60 \mathrm{~g}$. The frame, 3D printed on a Connex Objet 260, houses the rocker and servo, and is attached to the arm with a 2 inch Velcro strap. It has a curved bottom to rest comfortably on the arm, with a 3D printed rubber grip to keep it in place during use. The rocker has a radius of curvature of 20 $\mathrm{mm}$ with a $3 / 16$ inch $(5 \mathrm{~mm})$ neoprene foam strip to avoid slipping and increase comfort.

The axis of rotation for the rocker is set so that the contact point of the rocker has a $10 \mathrm{~mm}$ offset, $d$, from the bottom of the frame. This offset serves to create a normal force while reducing the tightness of the strap. The rocker is held in the frame by two shaft supports, and is driven by a digital servo (Futaba S3154), which is secured to the frame with two socket head screws and nuts (M1.6 x $0.35 \mathrm{~mm}$ ). Figure $3 \mathrm{~b}$ shows an image of the device on a subject's upper arm. The rocker rests in the neutral position shown in Figure $3 \mathrm{~b}$ when the hand is completely open, and rotates up to 60 degrees (Figure $3 \mathrm{a}$ ) according to the reference signal, which for the case study considered in this paper is the encoder of the motor of the Pisa/IIT SoftHand. The skin is stretched as the rocker rotates, for a maximum displacement of about $10.5 \mathrm{~mm}$. This maximum value of the rocker displacement angle is chosen to avoid slipping on the skin and is kept constant for all subjects. Commanded angles for the servo are obtained by linearly mapping the hand encoder reading, which assumes values within a certain range. These values were estimated from a preliminary test observing encoder readings during a series of ten close-open cycles of the hand from the resting open position. We refer the reader to [20] for more details.

\section{ABLE BOdIED STUdies: METHODS}

A total of 44 able-bodied subjects took part in the experiments described in this section. Of these, 14 (age $25.9 \pm 0.3$, 6 females) took part in a method of constant stimuli experiment to evaluate Just Noticeable Difference (JND) and Point of Subjective Equivalence (PSE); another 14 took part in a cue persistence experiment (age $23.5 \pm 0.5$ years, 4 female); and the remaining 16 (age 23.0 $\pm 0.3,6$ female) took part in an active size discrimination task. In the cue persistence and active size discrimination task half of the subjects were under the Haptic Feedback (HF) condition, and the half in the No Haptic Feedback (NHF) condition; male and female participants were distributed equally. All participants were right handed, and did not suffer from any physical or cognitive impairment, which could interfere with their ability to follow the instructions of the study, nor any pathology that could affect tactile sensation or muscular activity of the forearm. The methods and procedures described in this paper were carried out in accordance with the recommendations of the Institutional Review Board of Rice University with written informed consent obtained from all subjects.

\subsection{Just Noticeable Difference}

We evaluated the Just Noticeable Difference and Point of Subjective Equivalence for the Rice Haptic Rocker by using the method of constant stimuli in a perceptual experiment [48]. Each participant was wearing the Rice Haptic Rocker, was blindfolded and wore headphones with pink noise to cover noise from the servo motor. A series of coupled stimuli was delivered, with the first always being the Rocker commanded position of 45 degrees and the second being randomly taken from a list of equally spaced commanded positions between 37.8 and 52.2 degrees. The highest and lowest values were chosen to span reasonable values provided by the Rocker during grasp, and were coherent with the values spanned during the active size discrimination experiment. Participants reported, after each trial, which of the two stimuli they thought was higher.

We modeled the responses using a Generalized Linear Mixed Model (GLMM) approach, as described by Moscatelli et al. in [49], which when applied to psychophysics has the advantage of being able to treat data from multiple subjects as a whole [50]. Additionally, results from this test were used to evaluate the results in the active size discrimination task, as detailed later, and will thus be presented together with them.

\subsection{Cue persistence task}

The focus of this experiment was investigating what happens when the haptic task is not present in isolation, but is accompanied by a parallel task of a different nature. This provides insight on how effective the haptic feedback is under a condition of higher cognitive burden, as happens in everyday life when people split their attention to perform multiple parallel tasks, while still using proprioceptive information.
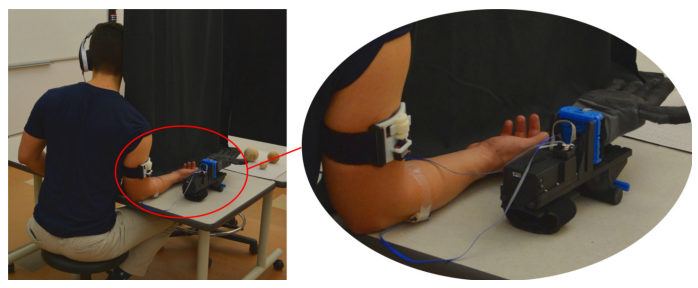

Fig. 4: Experimental setup for the cue persistence and passive size discrimination tasks.

In [24], vibrotactile feedback was used to convey information on the opening of a virtual hand. A parallel auditory task was used as distraction, and participants had to match the virtual hand opening to a target while performing operations on series of beeps. Taking inspiration from this work, we present an experiment where subjects close the hand, complete an auditory task intended to cause them to loose focus on the skin stretch task, and afterward discriminate whether they hold a sphere or not, based on the amount of stretch that has been held by the Rocker. Our objective 
was to study if the feedback can be intuitively understood in the presence of another task. The experimental set up and training phases in this experiment were the same presented for the passive size discrimination task described in [20], shown in Figure 4.

\begin{tabular}{|l|l|}
\multicolumn{1}{|c|}{ Haptic Task } & \multicolumn{1}{c|}{ Acoustic task } \\
\hline 30 repetitions; & $\begin{array}{l}\text { Is triggered immediately after } \\
\text { the hand closes and lasts 22 sec- } \\
\text { onds; } \\
20 \text { beeps are played for each rep- } \\
\text { etition; } \\
\text { The sphere is placed for 15 } \\
\text { times, while for the other half } \\
\text { the SoftHand is empty (random- } \\
\text { ized sequence); }\end{array}$ \\
$\begin{array}{l}\text { After the acoustic task subjects } \\
\text { have to tell if the sphere is in the } \\
\text { SoftHand or not. }\end{array}$ & $\begin{array}{l}\text { subjects are asked to count; } \\
\text { Subjects are told that this is the } \\
\text { important task that they should } \\
\text { focus on. }\end{array}$ \\
\end{tabular}

TABLE 1: Outline for the cue persistence with distraction task.

The testing phase lasted on average around 15 minutes. Participants completed 30 trials, each consisting of a haptic and auditory task. In each trial, participants were presented with one of two grasping conditions, an empty hand or a 2.5 inch $(63.5 \mathrm{~mm})$ wooden sphere, which represented the intermediate stimulus that was given in [20] for the passive size discrimination task and was chosen to provide an average level of difficulty. The test began with subjects commanding the closure of the hand after the stimulus was provided. The encoder measurement was used to detect the instant when the hand stopped, either wrapping around the sphere or closing while empty. As soon as this was detected, the auditory task began automatically, where they listened to a segment of 20 beeps in the headphones. Participants were told that this was the critical task demanding their focus and attention. The beeps were on two distinct levels of volume ("loud" or "soft"); the number of loud beeps and their order in the segment were randomly generated. Participants were asked to count the number of loud beeps, and report it at the end of the task. After completing the auditory task, subjects were asked whether they were holding a sphere. Those in the HF condition relied on the sensation from the Rocker, whereas those in the NHF condition could only guess. A statistical analysis was performed to test for a reliable difference between the HF and NHF condition performances, for both the haptic task and acoustic task. Normality of the data was evaluated with the Shapiro-Wilk test, to decide between using a t-test or the non-parametric Wilcoxon-Mann-Whitney test.

\subsection{Active size discrimination task}

Both the size discrimination task presented in [20] and the distraction task presented in the previous section were passive, in the sense that the participants were not moving the SoftHand to reach for an object, but were instead handed the spheres by the experimenter. This was necessary in order to have controlled experiments and limit the presence of confounding factors; however, it is very distant from a real case scenario where an upper limb prosthesis user is reaching out to grasp an object, especially when a multi-d.o.f. hand is considered. Indeed, while the former condition will lead to a low variability in finger configurations when grasping the same object, the latter yields a much increased variability, thus making the process of conveying information on

hand opening more challenging, as we briefly discussed in Section 2. With this motivation in mind, here we describe an experiment that aims to evaluate what happens when our setup is tested in an active size discrimination task.

In order to have an experimental setup that is closer to a real world scenario, we would like to have participants reach and grasp an object while moving the SoftHand themselves. However, to be able to evaluate the effectiveness of haptic feedback in particular, we cannot simply have them perform the task with their vision unimpaired, since it is well known that vision dominates over the sense of touch for tasks such as size discrimination, even when compared to able-bodied hands [51]. At the same time, if participants are blindfolded to completely block their sense of vision, it would be very difficult for them to complete any grasping task when relying only on the proprioceptive feedback from the Rice Haptic Rocker, let alone when doing the task under the no haptic feedback condition as part of the control group. For this reason, in the experiments described here participants had their vision partially impaired using glasses covered with frosted paper. Subjects retain enough visual information to locate objects in the workspace, but lack the visual fidelity necessary to conduct the size discrimination task based on visual feedback alone.

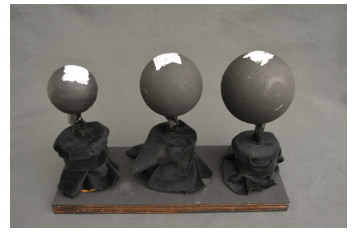

(a) Spheres on the stands.

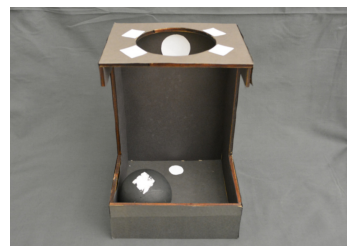

(c) Box used in the experiments.

Fig. 5: Active size discrimination task: experimental setup.

Figure 5 shows the experimental setup. Three boxes as in Figure $5 c$ were prepared, with a hole on the top of each. The boxes were covered with black paper, and smaller pieces of white paper were placed around the hole. At the same time, three different sizes $(2,2.5$ and 3 inches, corresponding to $50.8,63.5$ and $76.2 \mathrm{~mm}$ respectively) of polystyrene spheres were painted black, leaving a $1 \mathrm{~cm} \times 1 \mathrm{~cm}$ white square on each sphere. The white regions on objects were designed to be identical for each object so as to assist with locating the objects given the reduced visual fidelity from the frosted glasses, while still retaining the necessity of proprioceptive cues to complete the task. The spheres had a mass of 1.7, 3 and 5 grams. Since the overall weight of the SoftHand and EMG handle interface was $1.2 \mathrm{Kg}$, the weight difference was not enough to make weight-based discrimination possible [48]. Furthermore, stands with different heights were prepared for each sphere, and a small hole was drilled underneath, on the opposite side of the white square, to place the spheres on them easily (Figure 5a). The stands were designed so that all spheres would appear to have 
the same height from the bottom of the stand to the top of the sphere when placed in front of the subject. For the experiment, the three boxes were placed on a table covered with black fabric, and a black curtain was placed behind the table. A base was attached to the table with two slots designed to snap the stands in place. Finally, a red stop push button was connected to two of the Arduino analog pins, and the electric signal associated with it was monitored through MATLAB during the data acquisition; a mobile phone screen was also connected to MATLAB and used by the experimenter as a visual check of the status of the button.

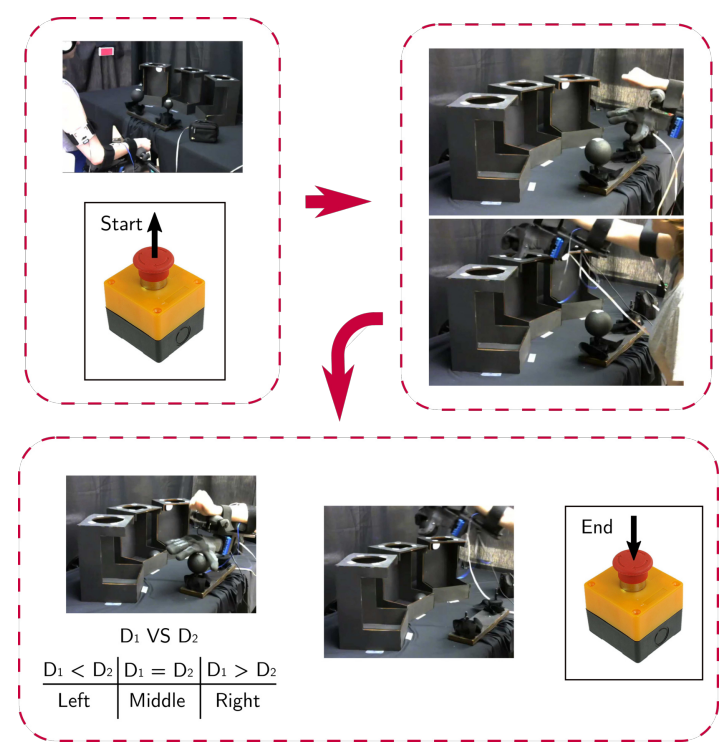

Fig. 6: Active size discrimination task. Participants hold the rest position with the SoftHand leaning on their right leg, until the red button is lifted. After hearing the go cue they lift the sphere to the right and place it in the box in the middle, then they lift the second sphere, estimate its diameter $D_{2}$ respect to the previous $D_{1}$, and drop it in one of the boxes according to the estimated size difference. The experimenter pushes down the red button immediately after the sphere is dropped; this is used to measure how much time was necessary to complete the task.

During the experiment, participants wore, in addition to the frosted paper glasses, noise canceling headphones, and pink noise was played through them to obscure the noise from the SoftHand motor. The headphones were also used to communicate to the participant the beginning of the task. The red button was used for this purpose; when lifted, it would cause a train whistle sound to play through the headphones, which was used as a go signal. At the beginning of each trial, two randomly selected spheres (which could also have the same size) were placed on the bases. After the go signal was played, participants reached for the sphere to the right, lifted it, and placed it in the box in the center, dropping it through the hole. They then reached for the sphere to the left, lifted it and placed it in one of the other boxes according to its size. In the instant when they dropped the second sphere in one of the boxes the experimenter pressed the red button; this played a gong sound through the headphones to signal that the participant could go back to the rest position, and recorded the time from the beginning of the trial. Then, the next set of stands for the following trials was prepared and placed.
To ensure that the subject could not see the size of the spheres as they were being placed, the experimenter wore black gloves through the whole experiment. Each pair of spheres was presented two times (with inverted positions) in a randomized sequence, for a total of 12 trials for each subject; the testing phase lasted approximately 20 minutes on average.

Before the experiment, each participant was instructed about the task. They were told that both accuracy and time of execution were going to be evaluated, and were allowed to practice two times to ensure that they understood the instructions and that they were able to complete the task despite the visual impairment. They were also asked if they were able to discriminate sphere size through vision, to which every participant reported a negative answer. After the experiment was finished, they were asked to complete a Likert-style survey.

Statistical analysis was performed to better evaluate the results, by first testing for normality with a Shapiro-Wilk test and then running a t-test or a non-parametric test when appropriate. This was done for both performance and time. A comparison based on the JND results was also done, by identifying three possible correct answers that would derive from a certain pairing of angles commanded to the Rocker and the consequent stimulus delivered to the subject: (i) $\left|\theta_{2}-\theta_{1}\right|<\mathrm{JND}$, i.e. there is no perceivable difference, so the two spheres should have been classified as having the same size; (ii) $\theta_{2}-\theta_{1}>$ JND, which means that the stimulus from the second sphere should have been perceived as larger, i.e. the hand closed more, leading to the second sphere being judged smaller; (iii) $\theta_{1}-\theta_{2}>$ JND, which should have lead to the second sphere being judged larger. As we will show in section 5, this is not equivalent to comparing accuracy based on spheres size because of variability in how the hand wraps around the object.

\section{ABLE BODIED STUdIES: RESULTS}

\subsection{Cue Persistence}

Figure 7 shows an overall view of performances for the haptic and acoustic task. Figure 7a represents correct and wrong answers for each trial of the haptic task with white and black squares respectively, while Figure $7 \mathrm{~b}$ uses a colormap to describe the difference between the number of high volume beeps as they were counted $\left(n_{c}\right)$ and the real number of higher volume beeps $\left(n_{r}\right)$. Inspection of the tables suggests a better performance for the HF condition in the haptic task, and a comparable performance in the acoustic task.

Figure 8 shows the discrimination accuracy for each subject in the HF condition, with light blue bars, and the NHF condition, with dark gray bars. Within each experimental group, the average accuracy was $76.2 \pm 7.1 \%$ for those the HF condition, and $48.6 \pm 7.9 \%$ in the NHF condition, showing better performance in the HF group and close agreement in the NHF group with the $50 \%$ chance level. The Shapiro-Wilk test showed that the assumption of normality is met for both the HF $(p=.46)$ and NHF $(p=.43)$ conditions, allowing to use a t-test for further analysis. There is a significant difference between the HF and NHF conditions $(p<.001)$.

The percentage of trials with the correct count for each subject are shown in Figure 9, with blue and red bars for participants in the HF and NHF conditions, respectively. 


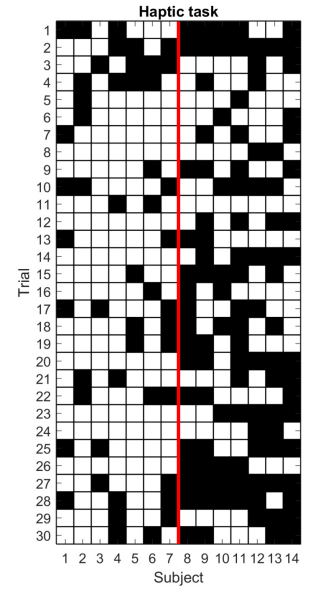

(a) Performance overview for the haptic task. White squares represent correct answers, while black squares represent incorrect answers.

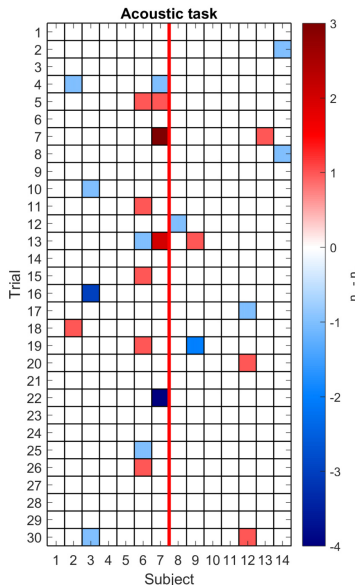

(b) Performance overview for the acoustic task. $n_{c}$ is the number of higher volume beeps counted, $n_{r}$ is the real number of loud beeps.
Fig. 7: Haptic and auditory task performance in the cue persistence with distraction task. Subjects 1-7 were under the Haptic Feedback condition, subjects 8-14 under the No Haptic Feedback condition. No evidence of correlation was found between haptic and acoustic performances $(-0.04$ correlation coefficient, $p>.56$ ).

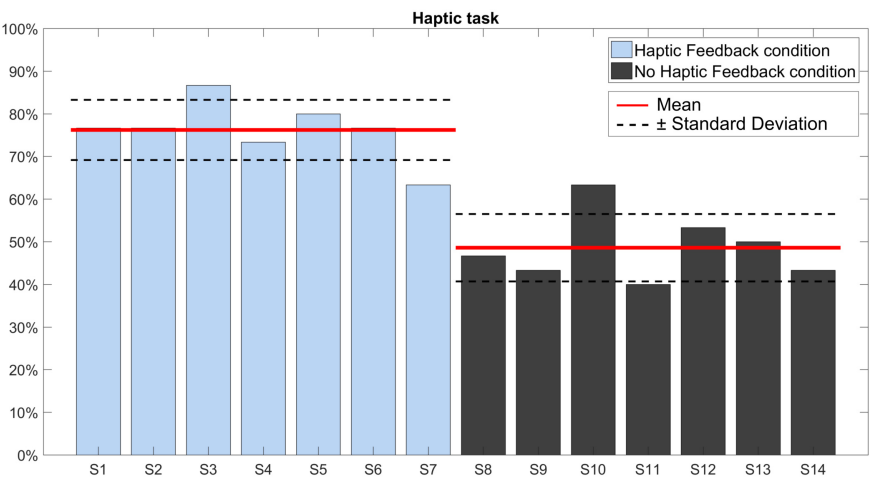

Fig. 8: Discrimination accuracy to detect presence of a sphere after an auditory distraction task.

The average accuracy for subjects under the HF condition was $91.9 \pm 9.20 \%$, and $95.7 \pm 3.7 \%$ for those in the NHF condition. The Shapiro-Wilk test for normality is not significant for the HF $(\mathrm{p}=.18)$ or the NHF $(\mathrm{p}=.48)$ conditions. Since the assumption of normality is met, a t-test is used for the comparison. There is no significant effect of the presence of haptic feedback $(p<.34)$.

\subsection{Just Noticeable Difference and Active size discrim- ination task}

Figure 10 shows the discrimination accuracy for each subject in the active size discrimination task. It can be seen that, while performance under the haptic feedback (HF) condition is slightly better than performance under the no haptic feedback (NHF) condition, the difference is not as evident as it was in the passive size discrimination experiments; variability among subjects also appears to be higher. Average accuracies are $45.83 \pm 14.77 \%$ for the HF condition

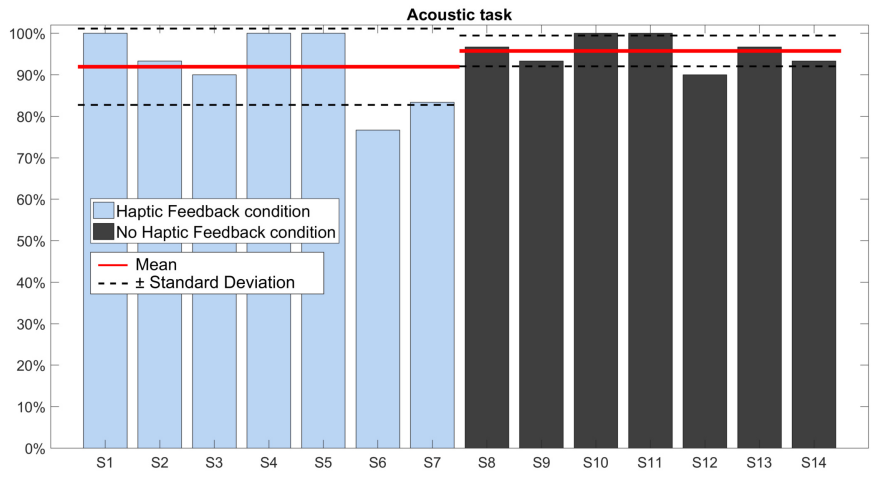

Fig. 9: Percentage of trials with correctly reported number of loud beeps during the auditory task.

and $36.46 \pm 21.33 \%$ for the NHF condition, with a chance level of $33 \%$. Figure 11 shows a comparison of pairwise size discrimination accuracy; in general it can be seen that performance is slightly higher for the HF condition.

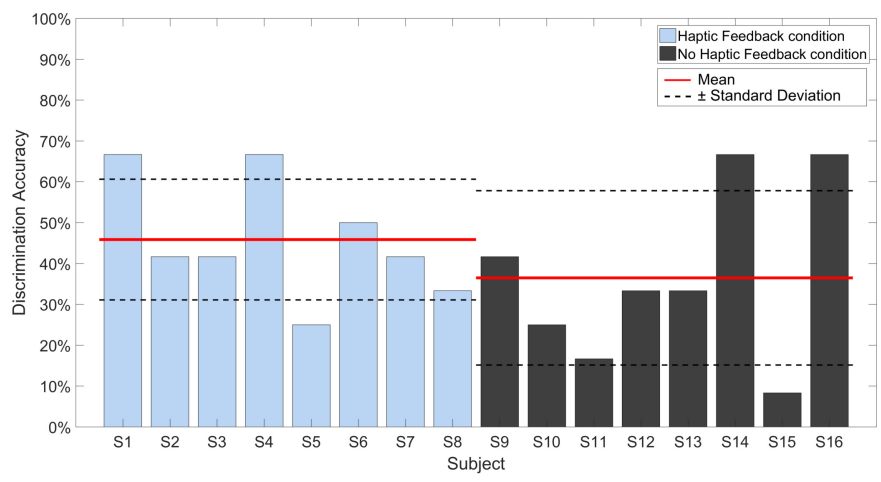

Fig. 10: Subjectwise accuracy for the active size discrimination task.

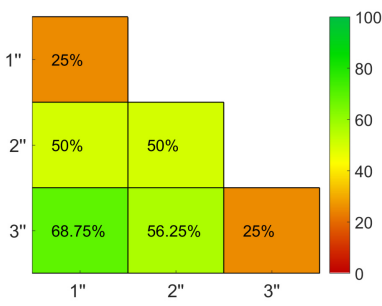

(a) HF condition.

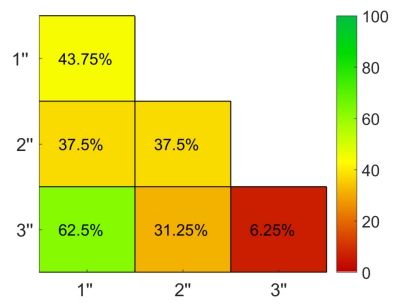

(b) NHF condition.
Fig. 11: Pairwise size discrimination accuracy. Each square represents the percentage of accurate identification for a pair of stimuli, independently from the order in which they were presented .

The Shapiro-Wilk test for normality showed that both discrimination accuracy and time were not significantly different from a normal distribution $(\mathrm{p}=.33 \mathrm{HF}, .40 \mathrm{NHF}$ and $\mathrm{p}=.33 \mathrm{HF}, .52 \mathrm{NHF}$ respectively). A t-test was used for the comparison, which did not show a significant effect for the presence of haptic feedback $(p<.33$ for the discrimination accuracy and $\mathrm{p}<.64$ for the time).

Table 2 shows the results for the Likert style survey that the participants took at the end of the experiment. Results 
are coherent with those found in [20] for what concerns subjective evaluation of the proposed setup (Q1-Q4 and Q7$\mathrm{Q} 10)$, and provide indication that the experimental setup was sound. In particular, for what concerns visual impairment (Q11 and Q12,) all participants reported being unable to discriminate spheres by size based on looking at the spheres through the frosted paper glasses.
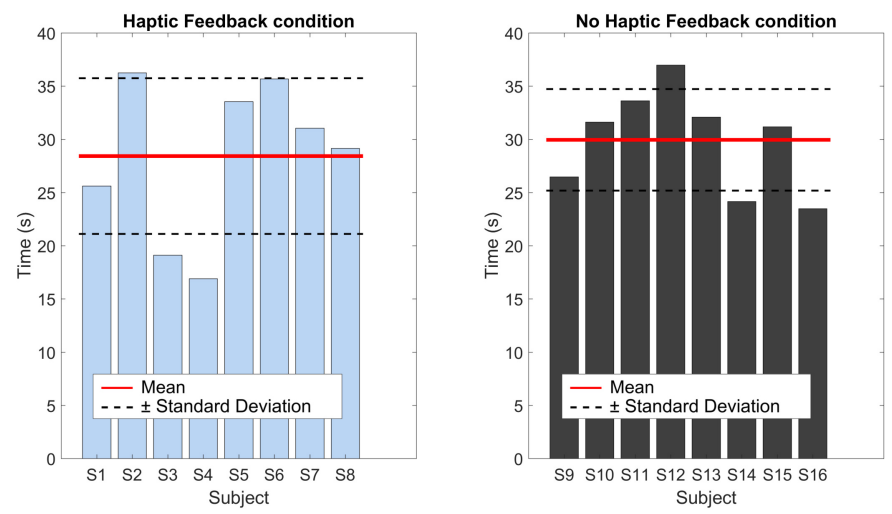

Fig. 12: Subjectwise mean time for the active task.
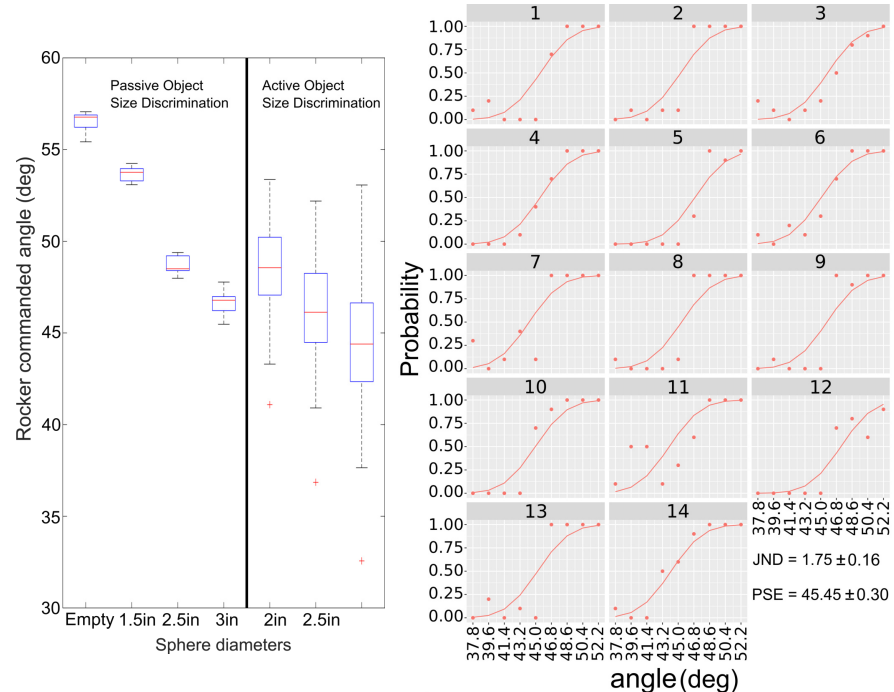

(a) Rocker angle for pas- (b) Psychometric curves for the sive and active task.

method of constant stimuli. JND and PSE with their standard errors are also reported.

Fig. 13: Evaluation of the Rocker commanded angle in the active size discrimination task and psychometric analysis.

Since the Rice Haptic Rocker proved to be effective in the previous experiments, the fact that we failed to obtain a statistically significant difference between the two can be surprising. The motivation behind this finding lies in an increased variability of the finger positioning of the SoftHand in the active grasp task. Figure 13a shows a box plot of the commanded angle for the Rocker for both the new active task and the passive task that was presented in our previous conference publication. It can be seen that, while in the passive task the commanded angles were different even for spheres that are close in size, in the active task there is a much greater variability, which could make the spheres difficult to distinguish. In other words, the haptic feedback from the Rocker remains reliable in providing information to the subjects, but this information could be misleading for this particular task because of the compliance of the hand.

To investigate this further we compared the results obtained under the HF and NHF conditions by taking into account the outcome of the Just Noticeable Difference experiment, as described in the last paragraph of Section 4. Figure $13 \mathrm{~b}$ shows the fitted psychometric curves and estimated values of JND and PSE. The JND is in line with values that were found for rotational skin stretch by Bark in [52] (between 2 and 4 degrees, as reported at page 106). Chinello et al. in [37] evaluated JND for their multi-rocker wrist device under a large amount of different conditions, varying force applied and position on the forearm, and found a JND between 3.5 and 1.5 degrees. The values found for the Rocker appear then to be in line with those found for other skin stretch devices in the state of art.

Reevaluating the performance according to the reference described in the last paragraph of Section 4 (i.e., scoring answers based on the Rocker commanded angle instead of the spheres size) leads to a mean accuracy of $52.08 \pm 13.17 \%$ for the HF and of $31.25 \pm 18.23 \%$ for the NHF condition, which also results significant at a $5 \%$ level when a t-test is done $(p<.022)$.

\section{AbLe BOdIED STUdies: DISCUSSION}

\subsection{Cue persistence task}

In this experiment, the Rice Haptic Rocker was successfully able to convey a proprioceptive cue even after attention was drawn to an unrelated task. Participants were able to distinguish the presence or absence of a sphere after a distraction. This persistent proprioceptive information is important for tasks such as holding a cup while talking to someone or looking at a screen, or holding an object in presence of distractions in general. The results would be less substantial if the participants with the Rocker present prioritized the haptic sensations over the auditory task, and the distraction was not drawing their full attention; this is why participants were instructed to focus their attention on the acoustic task. Performance in the acoustic task was not significantly different for the HF and NHF conditions, suggesting that the haptic task was not dominant over the acoustic task for those in the haptic feedback group.

\subsection{Just Noticeable Difference and Active size discrim- ination}

Looking at the results of the active size task, and its reevaluation based on the JND findings, it seems that the Rocker itself is effective in conveying feedback, but the increased variability of the SoftHand poses made conveying proprioceptive information challenging for the chosen task. In the passive size discrimination task, this problem was avoided by using spheres with larger differences in diameter (1.5, 2.5 , and 3 inches) compared to those used in the active size discrimination experiment presented in this paper $(2$, 2.5 and 3 inches). Preliminary tests had shown that using very small or very large spheres caused the participants to have problems doing the task successfully, which reduced the range of possible spheres that could be used. The inclusion of additional encoders and sensing elements could provide more complete measurements during hand closure; however, as we discussed in Section 2, it would also increase 


\begin{tabular}{cc} 
NHF & p-value \\
\hline N/A & N/A \\
N/A & N/A \\
N/A & N/A \\
N/A & N/A \\
$1.00(1.50 ; 3.00)$ & 1.00 \\
$5.00(6.50 ; 7.00)$ & 0.57 \\
N/A & N/A \\
N/A & N/A \\
N/A & N/A \\
N/A & N/A \\
$6.00(7.00 ; 7.00)$ & 0.57 \\
$1.00(1.00 ; 2.00)$ & 0.93 \\
$7.00(7.00 ; 7.00)$ & 1.00 \\
$1.00(1.00 ; 1.00)$ & 1.00 \\
$3.50(5.50 ; 6.50)$ & 0.99 \\
$2.00(3.50 ; 5.00)$ & 0.64
\end{tabular}

TABLE 2: Results of the Likert scale survey. The central tendency of responses is summarized by using median with dispersion measured by IQR $\left(25^{\circ} \sim 75^{\circ}\right)$. P-values for common questions are also reported; no significant difference was found.

complexity of the system. Delivering information on hand opening in a simple, if coarse, way might be preferable to delivering more complete information in a way that makes the prosthesis fragile while also making the haptic feedback less intuitive, thus frustrating the user. In the following section we will show a pilot test that we did with a single upper limb prosthesis user, to evaluate usage in a clinical test based on daily life tasks.

\section{Preliminary eXPERIMENTS WITH AN UPPER LIMB PROSTHESIS USER.}

We conducted an evaluation of the Rice Haptic Rocker with one individual with upper limb loss (SA1, female, 33 years old). SA1 suffered an amputation below the elbow on her left arm, and is an experienced myoelectric prosthesis user. Her able-bodied right arm is also her dominant arm. Her myoelectric prosthesis includes an active wrist, which was maintained in the setup with the SoftHand ${ }^{1}$. SA1 took part in two experiments: an AM-ULA assessment with and without haptic feedback, and a passive size discrimination task with haptic feedback. The experiments were divided in two sessions. During the first session she performed the AM-ULA with haptic feedback, while during the second session she performed the AM-ULA without haptic feedback and the passive size discrimination task with haptic feedback. Before each session, the same calibration and training procedures described for the experiments with ablebodied participants were followed.

The AM-ULA, or Activities Measure for Upper Limb Amputees, is an evaluation procedure for upper limb prostheses that was introduced in [25], where it was also tested for interrater reliability. Based on the evaluation of performance during daily living activities, it is now one of the standard assessment procedures that are used in prosthetics [53]. The test consists of a series of tasks that are carried out in a single session, with an occupational therapist guiding the participant, providing indications on how the task should be done and evaluating performance through a score ( 0 to 4$)$ that is given for each task. The zero score corresponds to the individual's inability to complete the task, while scores 1 to 3 are given based on speed,

1. For this experiment, the prosthetic version of the Pisa/IIT SoftHand was used, which includes a standard Ottobock quick-wrist connection. For this reason it was possible to simply remove the myoelectric hand that she has for daily use and replace it with the SoftHand for the experiments presence of awkwardness and/or compensatory movement, skill with the prosthesis and use (or lack of thereof) of additional assistive devices. A score of 4 corresponds to performance comparable to the ability of an intact hand.

\begin{tabular}{clcc}
\multicolumn{2}{c}{ Task and description } & HF & NHF \\
\hline 1 & Brush Hair & 3 & 3 \\
2 & Button shirt & 2 & 2 \\
3 & Zip jacket & 1 & 1 \\
4 & Put on socks & $\mathbf{2}$ & 1 \\
5 & Tie shoelaces & 1 & 1 \\
6 & Drink from a paper cup & $\mathbf{2}$ & 1 \\
7 & Use a fork (unilateral) & 1 & 1 \\
8 & Use a spoon (unilateral) & 1 & 1 \\
9 & Pour from a 12 oz can & $\mathbf{2}$ & 1 \\
10 & Write the word "LETTER" legibly (unilateral) & 1 & 1 \\
11 & Turn a round door knob & 1 & $\mathbf{2}$ \\
12 & Fold a bath towel & 3 & 3 \\
13 & Reach overhead (unilateral) & 1 & 1
\end{tabular}

TABLE 3: AM-ULA results. Scores can be 0 to 4 , where 0 corresponds to inability to complete the task and 4 corresponds to the ability of a able-bodied human hand.

This test, contrary to other popular assessments (e.g., box and blocks, nine peg hole test, Southampton hand assessment procedure etc.), does not focus heavily on speed. This characteristic made the AM-ULA ideal for our case, since it was expected that proprioceptive feedback might be useful for what concerns quality of grasp and manipulation more than for raw speed of task completion. Not all tasks of the AM-ULA were conducted in our study, since some of them would not have been feasible for the participant because of the experimental setup and integration of the Rice Haptic Rocker with the Pisa/IIT SoftHand (e.g., putting a shirt on or removing it would have not been possible without taking off the Rocker, because of the wiring). For this reason, the occupational therapist that was involved in the study chose a subset of tasks that were deemed to be feasible given the presence of our experimental hardware.

Table 3 shows the tasks that were selected as well as the score for each, with higher scores highlighted in bold where there was a difference between the two conditions. The scores are on average better for the HF condition, which is encouraging especially since the NHF condition was run after the HF condition, and thus in the NHF condition, the participant had already had a chance to familiarize themselves with the setup. This was particularly evident for task number 11 . When trying this task for the first time, the participant was unsure about how to approach the problem, and it took some time for her to find the right strategy to 
accomplish the task. By the second session, she was already familiar with the task and was able to accomplish it in a faster way. The proprioceptive feedback seemed to be effective for tasks were the fingers were occluded from view, i.e. Task 4 which involved putting on socks, and Tasks 6 and 9 which involved grasping (approximately) cylindrical objects and doing a task with them. SA1 also reported that the haptic feedback made her feel more comfortable during Task 3, even though this was not reflected in a difference between the scores.

At the end of the second session, a passive size discrimination task was also administered. Since SA1 was using the prosthesis with the non-dominant arm, the aim was to investigate if the haptic feedback was more effective on the prosthesis side or on the non-dominant arm. Because of time constraints, a shortened version of the passive size discrimination that was previously administered with ablebodied subjects was performed, using the empty hand, and only the 2 and 3 inch diameter spheres. The test was repeated two times, once with the Rice Haptic Rocker above the elbow on the (left) prosthetic side and once with the Rocker on the (right) contralateral but dominant side.
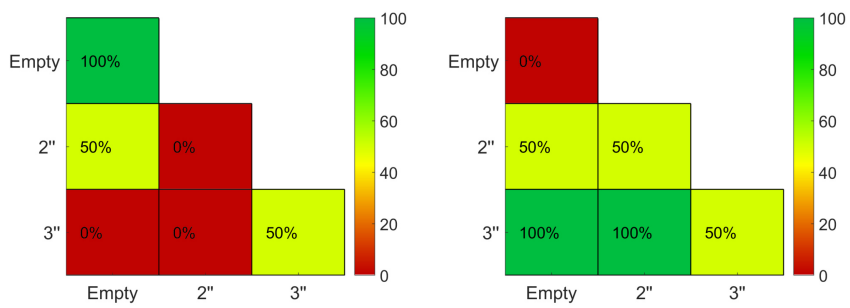

(a) Result for the test done on (b) Result for the test done on the left arm (prosthesis side). the right arm (dominant arm). Total accuracy 6/12 (50.0\%) Total accuracy $7 / 12$ (58.3\%)

Fig. 14: Pairwise accuracy in the size discrimination task done with SA1. Each square represents the percentage of accurate identification for a pair of stimuli, independently from the order in which they were presented. Note that these results are for one subject, i.e. 2 trials for each size pair.

Figure 14 shows the pairwise results. Interestingly, performance was slightly better for the contralateral side, perhaps owing to the fact that it was the subject's dominant arm; in both cases the observed accuracy was higher than the $33.3 \%$ chance level ( 1 on 3 chance of randomly guessing if the sphere was smaller, larger or the same size). The subject also reported a preference for having the haptic feedback on her right arm. Of course, since the test was conducted with only one participant, no strong conclusions can be drawn; however, this result opens up the possibility of evaluating the utility of contralateral haptic feedback in upper limb prosthesis users who have lost their nondominant hand.

\section{Conclusion}

In this paper we presented a one-d.o.f. wearable haptic feedback as a solution for conveying information on the opening (i.e., proprioception) of a multi-d.o.f., compliant prosthetic hand. More specifically, we discussed the design and evaluation of the Rice Haptic Rocker, which uses skin stretch to convey proprioceptive information in conjunction with the prosthetic version of the Pisa/IIT SoftHand, with different evaluation tests with able-bodied subjects and preliminary tests with an amputee.

For what concerns able-bodied subjects, two tests were performed: the first was a distraction task, where an acoustic task was ran in parallel with the haptic task to verify how robust the haptic feedback is to disturbance. Results of this test were positive $(76.2 \%$ accuracy under the haptic feedback condition vs $48.6 \%$ for the no haptic feedback condition, with a $50 \%$ chance level); the parallel acoustic task had an accuracy greater than $90 \%$ under both conditions, with no significant difference being found between the two conditions for this task. This is indication that the increase in the cognitive load caused by the haptic feedback was limited and hence suggests a promising usage of the haptic feedback in real life settings, where users' attention is shared across different tasks.

The second test was an active size discrimination task, which aimed to evaluate the haptic feedback in a task where the hand was being moved freely by the participants, to better evaluate the challenges that come with conveying proprioceptive feedback for a multi-d.o.f. hand under such conditions. The haptic feedback did not show statistically significant improvement in performance for what concerns the size of the spheres; however, further evaluation done by taking into account the compliance of the hand showed that this was due to the increased variability in the pose of the single fingers around the objects grasped, and not because the Rocker was not effective. In this sense, this experiment provided some interesting insight in the challenging task of conveying proprioceptive feedback for a multi-d.o.f., compliant prosthetic hand in a real world scenario.

Finally, the test with a prosthesis user included the clinical test AM-ULA and a passive size discrimination test. The AM-ULA showed a better performance when using the Rice Haptic Rocker as feedback device, despite the fact that the no haptic feedback condition was run in the second session (when the subject was already familiary with the SoftHand and the tasks proposed). The passive size discrimination task showed a $50 \%$ accuracy when using haptic feedback on the (left) prosthetic hand and $58 \%$ when using it on the (right) dominant able-bodied arm, with a $33 \%$ chance level; this could be caused by the fact that the subject was right handed.

In conclusion, the proposed approach opens interesting paths for a real-world application. The active size discrimination task shows that it is not possible to convey precise information on the level of opening of each fingertip for a compliant, multi-d.o.f. hand when using a single d.o.f. for haptic feedback; however, the participants were able to successfully identify the overall level of opening of the hand as measured by the motor encoder. In other words, while users were unable to obtain information on the position of each digit from the haptic feedback, they were still able to receive an estimate of how open the hand was: this suggests a shift of focus to functional evaluation, to see how this coarse, but simple and easy to understand haptic feedback can improve the quality of life of prosthetic limb users. Future work will continue in this direction, building upon the preliminary AM-ULA results that we obtained with a prothesis user, with additional tests. Alternatives to the linear mapping from the SoftHand encoder and alternative placements for the Rocker will also be considered. 


\section{ACKNOWLEDGMENTS}

The authors gratefully acknowledge Kathryn Nedley for providing her expertise in the evaluation of the AM-ULA test, and Mikaela Juzswik for her unique contribution in the physical realization of some of the equipment used in the experiments. This work was partially supported by the European Commission project (Horizon 2020 research program) SOFTPRO (no. 688857) and by the NSF grant IIS1065497.

\section{RefERENCES}

[1] E. Biddiss, D. Beaton, and T. Chau, "Consumer design priorities for upper limb prosthetics," Disability and Rehabilitation: Assistive Technology, vol. 2, no. 6, pp. 346-357, 2007.

[2] S. Millstein, H. Heger, and G. Hunter, "Prosthetic use in adult upper limb amputees: a comparison of the body powered and electrically powered prostheses," Prosthetics and orthotics international, vol. 10, no. 1, pp. 27-34, 1986.

[3] S. L. Carey, D. J. Lura, M. J. Highsmith et al., "Differences in myoelectric and body-powered upper-limb prostheses: Systematic literature review," J Rehabil Res Dev, vol. 52, no. 3, p. 247, 2015.

[4] A. Chadwell, L. Kenney, S. Thies, A. Galpin, and J. Head, "The reality of myoelectric prostheses: Understanding what makes these devices difficult for some users to control," Frontiers in Neurorobotics, vol. 10, 2016.

[5] E. A. Biddiss and T. T. Chau, "Upper limb prosthesis use and abandonment: a survey of the last 25 years," Prosthetics and orthotics international, vol. 31, no. 3, pp. 236-257, 2007.

[6] C. Pylatiuk, S. Schulz, and L. Döderlein, "Results of an internet survey of myoelectric prosthetic hand users," Prosthetics and orthotics international, vol. 31, no. 4, pp. 362-370, 2007.

[7] S. Lewis, M. F. Russold, H. Dietl, and E. Kaniusas, "User demands for sensory feedback in upper extremity prostheses," in Medical Measurements and Applications Proceedings (MeMeA), 2012 IEEE International Symposium on. IEEE, 2012, pp. 1-4.

[8] H. H. Ehrsson, B. Rosén, A. Stockselius, C. Ragnö, P. Köhler, and G. Lundborg, "Upper limb amputees can be induced to experience a rubber hand as their own," Brain, vol. 131, no. 12, pp. 3443-3452, 2008.

[9] P. D. Marasco, K. Kim, J. E. Colgate, M. A. Peshkin, and T. A. Kuiken, "Robotic touch shifts perception of embodiment to a prosthesis in targeted reinnervation amputees," Brain, vol. 134, no. 3, pp. 747-758, 2011.

[10] A. Ninu, S. Dosen, S. Muceli, F. Rattay, H. Dietl, and D. Farina, "Closed-loop control of grasping with a myoelectric hand prosthesis: Which are the relevant feedback variables for force control?" Neural Systems and Rehabilitation Engineering, IEEE Transactions on, vol. 22, no. 5, pp. 1041-1052, 2014.

[11] H. J. Witteveen, E. A. Droog, J. S. Rietman, and P. H. Veltink, "Vibro-and electrotactile user feedback on hand opening for myoelectric forearm prostheses," Biomedical Engineering, IEEE Transactions on, vol. 59, no. 8, pp. 2219-2226, 2012.

[12] A. Ajoudani, S. B. Godfrey, M. Bianchi, M. G. Catalano, G. Grioli, N. Tsagarakis, and A. Bicchi, "Exploring teleimpedance and tactile feedback for intuitive control of the pisa/iit softhand," Haptics, IEEE Transactions on, vol. 7, no. 2, pp. 203-215, 2014.

[13] E. Treadway, B. Gillespie, D. Bolger, A. Blank, M. O'Malley, and A. Davis, "The role of auxiliary and referred haptic feedback in myoelectric control," in World Haptics Conference (WHC), 2015 IEEE. IEEE, 2015, pp. 13-18.

[14] Q. Fu and M. Santello, "improving fine control of grasping force during hand-object interactions for a soft synergy-inspired myoelectric prosthetic hand," Frontiers in neurorobotics, vol. 11, p. 71, 2018.

[15] K. Bark, J. Wheeler, P. Shull, J. Savall, and M. Cutkosky, "Rotational skin stretch feedback: A wearable haptic display for motion," Haptics, IEEE Transactions on, vol. 3, no. 3, pp. 166-176, 2010.

[16] K. Kim and J. Colgate, "Haptic feedback enhances grip force control of semg-controlled prosthetic hands in targeted reinnervation amputees," Neural Systems and Rehabilitation Engineering, IEEE Transactions on, vol. 20, no. 6, pp. 798-805, 2012.
[17] D. J. Atkins, D. C. Heard, and W. H. Donovan, "Epidemiologic overview of individuals with upper-limb loss and their reported research priorities." JPO: Journal of Prosthetics and Orthotics, vol. 8, no. 1, pp. 2-11, 1996.

[18] C. Piazza, G. Grioli, M. Catalano, and A. Bicchi, "A century of robotic hands," Annual Review of Control, Robotics, and Autonomous Systems, vol. (In Press), 2019.

[19] G. K. Patel, S. Dosen, C. Castellini, and D. Farina, "Multichannel electrotactile feedback for simultaneous and proportional myoelectric control," Journal of neural engineering, vol. 13, no. 5, p. 056015, 2016

[20] E. Battaglia, J. P. Clark, M. Bianchi, M. G. Catalano, A. Bicchi, and M. K. O'Malley, "The rice haptic rocker: skin stretch haptic feedback with the pisa/iit softhand," in World Haptics Conference (WHC), 2017 IEEE. IEEE, 2017, pp. 7-12.

[21] R. Christiansen, J. L. Contreras-Vidal, R. B. Gillespie, P. A. Shewokis, and M. K. O'Malley, "Vibrotactile feedback of pose error enhances myoelectric control of a prosthetic hand," in World Haptics Conference (WHC), 2013. IEEE, 2013, pp. 531-536.

[22] T. Pistohl, D. Joshi, G. Ganesh, A. Jackson, and K. Nazarpour, "Artificial proprioceptive feedback for myoelectric control," Neural Systems and Rehabilitation Engineering, IEEE Transactions on, vol. 23, no. 3, pp. 498-507, 2015.

[23] A. Blank, A. M. Okamura, and K. J. Kuchenbecker, "Identifying the role of proprioception in upper-limb prosthesis control: Studies on targeted motion," ACM Transactions on Applied Perception (TAP), vol. 7, no. 3, p. 15, 2010.

[24] H. Witteveen, L. de Rond, J. Rietman, and P. Veltink, "Handopening feedback for myoelectric forearm prostheses: performance in virtual grasping tasks influenced by different levels of distraction." Journal of rehabilitation research and development, vol. 49, no. 10, p. 1517, 2012.

[25] L. Resnik, L. Adams, M. Borgia, J. Delikat, R. Disla, C. Ebner, and L. S. Walters, "Development and evaluation of the activities measure for upper limb amputees," Archives of physical medicine and rehabilitation, vol. 94, no. 3, pp. 488-494, 2013.

[26] U. Proske and S. C. Gandevia, "The proprioceptive senses: their roles in signaling body shape, body position and movement, and muscle force," Physiological reviews, vol. 92, no. 4, pp. 1651-1697, 2012.

[27] — "The kinaesthetic senses," The Journal of physiology, vol. 587, no. 17, pp. 4139-4146, 2009.

[28] D. F. Collins, K. M. Refshauge, G. Todd, and S. C. Gandevia, "Cutaneous receptors contribute to kinesthesia at the index finger, elbow, and knee," Journal of Neurophysiology, vol. 94, no. 3, pp. 1699-1706, 2005.

[29] N. David, A. Newen, and K. Vogeley, "The "sense of agency" and its underlying cognitive and neural mechanisms," Consciousness and cognition, vol. 17, no. 2, pp. 523-534, 2008.

[30] C. D. Murray, "Embodiment and prosthetics," in Psychoprosthetics. Springer, 2008, pp. 119-129.

[31] J. S. Schofield, K. R. Evans, J. P. Carey, and J. S. Hebert, “Applications of sensory feedback in motorized upper extremity prosthesis: a review," Expert review of medical devices, vol. 11, no. 5, pp. 499511,2014

[32] C. J. Hasson and J. Manczurowsky, "Effects of kinematic vibrotactile feedback on learning to control a virtual prosthetic arm," Journal of neuroengineering and rehabilitation, vol. 12, no. 1, p. 1, 2015.

[33] M. D'Alonzo, S. Dosen, C. Cipriani, and D. Farina, “Hyve-hybrid vibro-electrotactile stimulation-is an efficient approach to multichannel sensory feedback," IEEE transactions on haptics, vol. 7, no. 2, pp. 181-190, 2014.

[34] K. Bark, J. W. Wheeler, S. Premakumar, and M. R. Cutkosky, "Comparison of skin stretch and vibrotactile stimulation for feedback of proprioceptive information," in Haptic interfaces for virtual environment and teleoperator systems, 2008. haptics 2008. symposium on. IEEE, 2008, pp. 71-78.

[35] J. Wheeler, K. Bark, J. Savall, and M. Cutkosky, "Investigation of rotational skin stretch for proprioceptive feedback with application to myoelectric systems," Neural Systems and Rehabilitation Engineering, IEEE Transactions on, vol. 18, no. 1, pp. 58-66, 2010.

[36] A. Akhtar, M. Nguyen, L. Wan, B. Boyce, P. Slade, and T. Bretl, "Passive mechanical skin stretch for multiple degree-of-freedom proprioception in a hand prosthesis," in International Conference 
on Human Haptic Sensing and Touch Enabled Computer Applications. Springer, 2014, pp. 120-128.

[37] F. Chinello, C. Pacchierotti, N. G. Tsagarakis, and D. Prattichizzo, "Design of a wearable skin stretch cutaneous device for the upper limb," in 2016 IEEE Haptics Symposium (HAPTICS). IEEE, 2016, pp. $14-20$

[38] R. Mann and S. Reimers, "Kinesthetic sensing for the emg controlled" boston arm"," IEEE Transactions on Man-Machine Systems, vol. 11, no. 1, pp. 110-115, 1970.

[39] M. Markovic, H. Karnal, B. Graimann, D. Farina, and S. Dosen, "Glimpse: Google glass interface for sensory feedback in myoelectric hand prostheses," Journal of neural engineering, vol. 14, no. 3, p. 036007, 2017.

[40] Ottobock, "Myoelectric speed hands," https://www.ottobockus. com/prosthetics/upper-limb-prosthetics/solution-overview/ myoelectric-devices-speedhands /.

[41] —, "Bebionic hand," https://www.ottobockus.com/ prosthetics/upper-limb-prosthetics/solution-overview/ bebionic-hand/.

[42] S. B. Godfrey, K. D. Zhao, A. Theuer, M. G. Catalano, M. Bianchi, R. Breighner, D. Bhaskaran, R. Lennon, G. Grioli, M. Santello et al., "The softhand pro: Functional evaluation of a novel, flexible, and robust myoelectric prosthesis," PloS one, vol. 13, no. 10, p. e0205653, 2018.

[43] M. Santello, M. Flanders, and J. F. Soechting, "Postural hand synergies for tool use," The Journal of Neuroscience, vol. 18, no. 23, pp. 10105-10115, 1998.

[44] P. H. Thakur, A. J. Bastian, and S. S. Hsiao, "Multidigit movement synergies of the human hand in an unconstrained haptic exploration task," The Journal of neuroscience, vol. 28 , no. 6, pp. 12711281, 2008.

[45] A. Bicchi, M. Gabiccini, and M. Santello, "Modelling natural and artificial hands with synergies," Philosophical Transactions of the Royal Society of London B: Biological Sciences, vol. 366, no. 1581, pp. 3153-3161, 2011.

[46] C. Della Santina, C. Piazza, G. M. Gasparri, M. Bonilla, M. G. Catalano, G. Grioli, M. Garabini, and A. Bicchi, "The quest for natural machine motion: An open platform to fast-prototyping articulated soft robots," IEEE Robotics \& Automation Magazine, vol. 24, no. 1, pp. 48-56, 2017.

[47] S. B. Godfrey, A. Ajoudani, M. G. Catalano, G. Grioli, and A. Bicchi, "A synergy-driven approach to a myoelectric hand," in $13 T H$ International Conference on Rehabilitation Robotics, June 24-26, 2013, Seattle, WA., 2013, pp. 1 - 6. [Online]. Available: 10.1109/ICORR.2013.6650377

[48] G. A. Gescheider, Psychophysics: the fundamentals. Psychology Press, 2013.

[49] A. Moscatelli, M. Mezzetti, and F. Lacquaniti, "Modeling psychophysical data at the population-level: the generalized linear mixed model," Journal of vision, vol. 12, no. 11, pp. 26-26, 2012.

[50] A. Moscatelli, M. Bianchi, A. Serio, A. Terekhov, V. Hayward, M. O. Ernst, and A. Bicchi, "The change in fingertip contact area as a novel proprioceptive cue," Current Biology, vol. 26, no. 9, pp. 11591163, 2016.

[51] M. O. Ernst and M. S. Banks, "Humans integrate visual and haptic information in a statistically optimal fashion," Nature, vol. 415, no. 6870, pp. 429-433, 2002.

[52] K. Y. J. Bark, Rotational skin stretch feedback: a new approach to wearable haptic display. Stanford University, 2009.

[53] A. W. Heinemann, L. Connelly, L. Ehrlich-Jones, and S. Fatone, "Outcome instruments for prosthetics: clinical applications," Physical Medicine and Rehabilitation Clinics, vol. 25, no. 1, pp. 179-198, 2014.

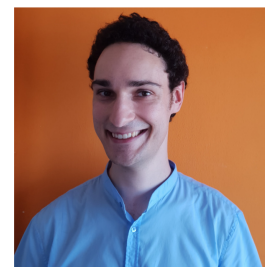

Edoardo Battaglia received his bachelor degree in Mechanical Engineering and his master degree in Robotics and Automation Engineering at the University of Pisa, in 2009 and 2013 respectively. He received his $\mathrm{PhD}$ in Information Engineering from University of Pisa in 2018. He is currently a Research Fellow at the Research Center "E. Piaggio" of the University of Pisa. His research interests include design and validation of haptic interfaces and mathematical modeling of the sense of touch and human manipulation.

of the sense of touch and human manipulation.
$1939-1412$ (c) 2018 IEEE. Personal use is permitted, but republication/redist

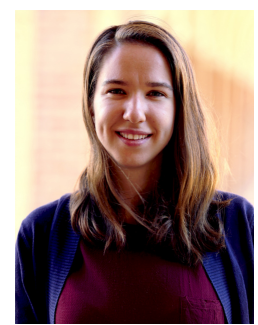

Janelle P. Clark received her bachelor degree in Mechanical Engineering at Clarkson University in 2015 and her master degree in Mechanical Engineering at Rice University in 2017. She is currently a PhD student working on haptic feedback for upper limb prostheses in the Mechatronics and Haptics Interfaces (MAHI) Lab at Rice University. Her research interests include coupled control of mechanical and electrical systems, rehabilitation robotics and haptic feedback as a surrogate sense of touch for amputees.

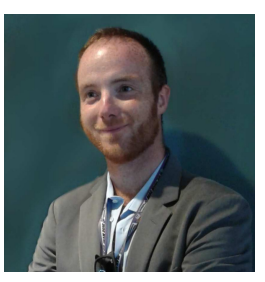

Matteo Bianchi received the B.S degree and the M.S cum laude in Biomedical Engineering from University of Pisa, Italy, in 2004 and 2007, respectively and the $\mathrm{PhD}$ in Robotics from University of Pisa in 2012. He is Assistant Professor at the University of Pisa. His research interests include haptic interface design, with applications in medical robotics and assistive/affective humanrobot interaction; human and robotic hands: optimal sensing and control; modelling of the sense of touch and human manipulation. He is editor of the book "Human and Robot Hands", Springer International Publishing.

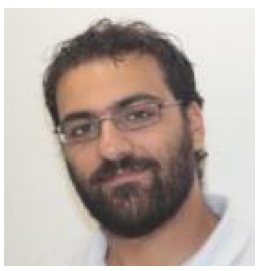

Manuel G. Catalano received the Laurea degree in mechanical engineering and the doctoral degree in robotics from the "E. Piaggio" Research Center at the University of Pisa. He is currently a Researcher at the Italian Institute of Technology and a collaborator of Centro $\mathrm{d}$ Ricerca "E. Piaggio" of the University of Pisa. His main research interests are in the design of Soft Robotics systems, Human Robot Interaction and Prosthetics. In 2014, he was the winner of the Georges Giralt PhD Award, the prestigious annual European award given for the best $\mathrm{PhD}$ thesis by euRobotics AISBL.

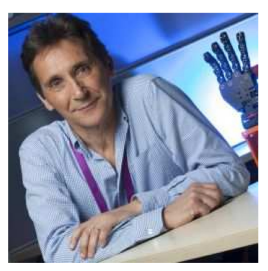

Antonio Bicchi is Senior Reseracher at IIT/ADVR and Rehab Technologies. He is also Professor of Robotics at the University of Pisa, and Adjunct Professor at Arizona State University. He graduated from the University of Bologna in 1988 and was a postdoc scholar at M.I.T. Artificial Intelligence lab between 1988 and 1991. His main research interests are in Robotics, Haptics, and Control Systems. He has published more than 400 papers on international journals, books, and refereed conferences. He has organized and co-chaired the first WorldHaptics Conference (2005), and Hybrid Systems: Computation and Control (2007). He is the recipient of several awards and honors.

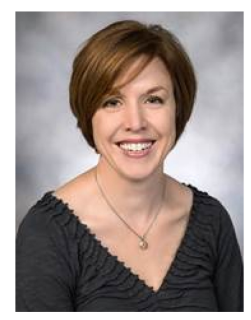

Marcia O'Malley received the B.S. degree from Purdue University, in 1996, and the M.S. and Ph.D. degrees from Vanderbilt University in 1999 and 2001, respectively, all in mechanical engineering. She is currently the Stanley C. Moore Professor in mechanical engineering, computer science, and electrical and computer engineering at Rice University, and directs the Mechatronics and Haptic Interfaces Laboratory. She is currently an Adjunct Faculty in the Departments of Physical Medicine and Rehabilitation at the Baylor College of Medicine, and the University of Texas Medical School at Houston, and the Director of Rehabilitation Engineering at TIRR-Memorial Hermann Hospital. Her research addresses issues that arise when humans physically interact with robotic systems, with a focus on training and rehabilitation in virtual environments. 